\title{
Purification of Peroxisomes Using a Density Barrier in a Swinging-Bucket Rotor
}

\author{
John M. Graham, Ph.D. \\ School of Biomolecular Sciences, Liverpool John Moores University, Office address: 34, \\ Meadway, Upton, Wirral CH49 6JQ \\ E-mail: john@jgrescon.fsbusiness.co.uk
}

Received March 7, 2002; Revised May 14, 2002; Accepted May 15, 2002; Published May 22, 2002

In iodixanol, peroxisomes are the densest organelle in the light mitochondrial fraction and are therefore easily separated from the other components (lysosomes, mitochondria, etc.) in a preformed isosmotic continuous gradient. Because of the large difference in density between peroxisomes and the next densest organelle (mitochondria), a density barrier is effective. The resolution of the peroxisomes is far superior than that in sucrose and, unlike in Percoll $\circledast$, there is no contamination from endoplasmic reticulum.

KEY WORDS: peroxisomes, light mitochondrial fraction, OptiPrep ${ }^{\mathrm{TM}}$, iodixanol, liver, density barrier

DOMAINS: protein trafficking, proteomics, cell biology, biochemistry, molecular biology, signaling, methods and protocols

METHOD TYPE: extraction, isolation, purification and separation

SUB METHOD TYPE: centrifugation

\section{INTRODUCTION}

Peroxisomes can be purified in iodixanol gradients in high yield (80-90\%) with no detectable contamination from any other organelle[1,2,3]. This is a property unique to iodixanol because the densities of other organelles, particularly that of mitochondria (approx. $\rho=1.14 \mathrm{~g} / \mathrm{ml}$ ) and endoplasmic reticulum (approx. $\rho=1.13 \mathrm{~g} / \mathrm{ml}$ ) are much lower than that of peroxisomes (approx. $\rho=1.18 \mathrm{~g} / \mathrm{ml}$ ). In $\mathrm{Nycodenz}{ }^{\circledR}$ and metrizamide, mitochondria have a significantly higher density (approx. $\rho=1.165 \mathrm{~g} / \mathrm{ml}$ ) than in iodixanol because only the latter can provide an iso-osmotic medium at densities above $\rho=1.15-1.16 \mathrm{~g} / \mathrm{ml}$. The density of peroxisomes on the other hand is 
relatively little affected by the type of iodinated density gradient medium because of their lack of an osmotic space. In Percoll both peroxisomes and endoplasmic reticulum have the same banding density and these two organelles cannot be resolved.

The protocol below is for the liver from a young adult male rat (wet weight approx. $10 \mathrm{~g}$ ), other tissues may require a different homogenization technique. It requires a simple iodixanol density barrier in a swinging-bucket rotor (see Note 1). Yields may be lower (approx. $40 \%$ of the catalase in the light mitochondrial fraction) than in a continuous gradient but the purity is as high.

\section{MATERIALS AND EQUIPMENT}

OptiPrep ${ }^{\mathrm{TM}}$

Working Solution (WS) of 54\% (w/v) iodixanol $(\rho=1.291 \mathrm{~g} / \mathrm{ml})$ : 9 vol of OptiPrep ${ }^{\mathrm{TM}}+1 \mathrm{vol}$ of $0.25 \mathrm{M}$ sucrose, $10 \mathrm{mM}$ EDTA, 1\% (v/v) ethanol, $200 \mathrm{mM}$ Hepes-NaOH, pH 7.4 (see Note 2)

Homogenization Medium (HM): $0.25 \mathrm{M}$ sucrose, $1 \mathrm{mM}$ EDTA, 0.1\% (v/v) ethanol, $20 \mathrm{mM}$ Hepes-NaOH, pH 7.4

High-speed centrifuge with a fixed-angle rotor (30-50 $\mathrm{ml}$ tubes) Ultracentrifuge with swinging-bucket rotor (tube capacity of approx. $17 \mathrm{ml}$ )

Potter-Elvehjem homogenizer (30-40 ml), clearance approx. $0.08 \mathrm{~mm}$

Wall-mounted, high-torque, thyristor-controlled electric motor

Dounce homogenizer (10 ml, loose-fitting, Wheaton Type B)

Syringe with metal cannula for underlayering

\section{METHOD}

1. Make up two dilutions of WS, containing $47 \%$ iodixanol $(\rho=1.257 \mathrm{~g} / \mathrm{ml})$ and $35 \%$ iodixanol (w/v) $(\rho=1.199 \mathrm{~g} / \mathrm{ml})$ by mixing WS and HM $(8.7+1.3$ and $6.48+3.52 \mathrm{v} / \mathrm{v}$, respectively). Keep these solutions, and carry out all subsequent operations, at $0-4^{\circ} \mathrm{C}$.

2. Mince the liver very finely with scissors and transfer to a Potter-Elvehjem (Teflon and glass) homogenizer with HM (use $10 \mathrm{ml}$ medium for every $2.5 \mathrm{~g}$ tissue). Homogenize using approx. 6 strokes of the pestle (500-700 rpm) (see Note 3).

3. Centrifuge the homogenate at $3000 g_{\mathrm{av}}$ in a fixed-angle rotor for $10 \mathrm{~min}$ to pellet the nuclei and heavy mitochondria (see Note 4).

4. Centrifuge the supernatant(s) at $17,000 g_{\mathrm{av}}$ for $10-15 \mathrm{~min}$ to produce a "light mitochondrial pellet".

5. Resuspend this pellet in approx. $8.0 \mathrm{ml}$ of $\mathrm{HM}$ (total volume) using a loose-fitting Dounce homogenizer (2-3 strokes of the pestle) and add an equal volume of $47 \%$ iodixanol. The refractive index of this suspension should be 1.3782; adjust to this value with the $47 \%$ iodixanol or WS if necessary. The density of this suspension is approx. $1.145 \mathrm{~g} / \mathrm{ml}$.

6. Transfer approx. $15 \mathrm{ml}$ of the suspension to a $17-$ to $20-\mathrm{ml}$ tube for a suitable swingingbucket rotor and underlayer with $1 \mathrm{ml}$ of the $35 \%$ iodixanol. Top up with tube with suspension or HM if necessary. Centrifuge at $110,000 \mathrm{~g}$ for $2 \mathrm{~h}$.

7. Aspirate and discard the majority of the liquid above the peroxisomes which band just above the original interface near the bottom of the tube, then harvest the band of peroxisomes. 
TABLE 1

Enzyme Analysis of Fractions

\begin{tabular}{|c|c|c|}
\hline Fraction & Catalase $^{1}$ & $\mathrm{SDH}^{1}$ \\
\hline Homogenate & 58.6 & nd \\
\hline Light mit pellet & 114.5 & 0.18 \\
\hline Barrier fraction & 1442.8 & 0.02 \\
\hline
\end{tabular}

\section{ANALYSIS}

Iodixanol does not significantly inhibit any enzyme so far tested. Standard spectrophotometric methods (carried out above $340 \mathrm{~nm}$ ), for measuring organelle enzyme markers can be performed directly on gradient fractions[4]. Protein can also be measured directly by any Coomassie bluebased method[4].

If it is necessary to remove the gradient medium, fractions can be diluted with an equal volume of buffer; pelleted at approx $30,000 g_{\mathrm{av}}$ for $10 \mathrm{~min}$ and resuspended in a suitable buffer (recoveries are $>90 \%$ ).

A typical enzyme analysis is shown in Table 1 (see Note 5).

\section{NOTES}

1. Other protocols for the isolation of peroxisomes require use either a preformed continuous density gradient[5] or a self-generated gradient in a vertical, or other short sedimentation path length rotor[6]. The simple density barrier technique described in this Protocol Article should provide organelles of similar purity but the yield is likely to be lower than that from the two continuous gradient systems.

2. Strategies for preparing gradient solutions for mammalian tissues are given in Ref. [7]. Protease inhibitors may be included in any or all of the media at the operator's discretion.

3. See Refs. $[8,9,10]$ for more information on homogenization of tissues and cells and differential centrifugation of an homogenate.

4. This pellet may be rehomogenized in HM and the centrifugation repeated.

5. The specific activity of catalase in the barrier fraction represents an approx. 25-fold purification over the homogenate and approx. $37 \%$ of the total catalase activity of the light mitochondrial pellet was recovered in the barrier fraction. Barrier techniques, while simple, are inevitably a compromise between purity and yield. Because of the density heterogeneity of all organelles it is impossible to choose a density for the suspending medium which is greater than that all of the mitochondria and less than that of all of the peroxisomes.

\section{ACKNOWLEDGEMENTS}

The author and TheScientific World wish to thank Axis-Shield PoC, AS, Oslo, Norway for their kind permission to adapt OptiPrep ${ }^{\mathrm{TM}}$ Application Sheet S10 in the preparation of this Protocol Article. 


\section{REFERENCES}

1. Graham J., Ford, T., and Rickwood, D. (1994) The preparation of subcellular organelles from mouse liver in self-generated gradients of iodixanol. Anal. Biochem. 220, 367-373.

2. Van Veldhoven, P.P., Baumgart, E., and Mannaerts, G.P. (1996) Iodixanol (OptiPrep), an improved density gradient medium for the isoosmotic isolation of rat liver peroxisomes. Anal. Biochem. 237, 17-23.

3. Gijsbers, S., Van der Hoeven, G., and Van Veldhoven, P.P. (2001) Subcellular study of sphingoid base phosphorylation in rat tissues: evidence for multiple sphingosine kinases. Biochim. Biophys. Acta 1532, $37-$ 50.

4. Ford, T., Graham, J., and Rickwood, D. (1994) Iodixanol: a non-ionic isoosmotic centrifugation medium for the formation of self-generated gradients. Anal. Biochem. 220, 360-366.

5. Graham, J.M. (2002) Purification of peroxisomes in a pre-formed iodixanol gradient in a fixed-angle rotor. TheScientificWorldJOURNAL 2, in press.

6. Graham, J.M. (2002) Purification of peroxisomes in a self-generated gradient. TheScientificWorldJOURNAL $\mathbf{2}$, in press.

7. Graham, J.M. (2002) OptiPrep ${ }^{\mathrm{TM}}$ density gradient solutions for mammalian organelles. TheScientificWorldJOURNAL 2, in press.

8. Graham, J.M. (2002) Homogenization of mammalian tissues. TheScientificWorldJOURNAL 2, in press.

9. Graham, J.M. (2002) Homogenization of mammalian cultured cells. TheScientificWorldJOURNAL 2, in press.

10. Graham, J.M. (2002) Preparation of crude subcellular fractions by differential centrifugation. TheScientificWorldJOURNAL 2, in press.

This article should be referenced as follows:

Graham, J.M. (2002) Purification of peroxisomes using a density barrier in a swinging-bucket rotor. TheScientificWorldJOURNAL 2, 1400-1403. 

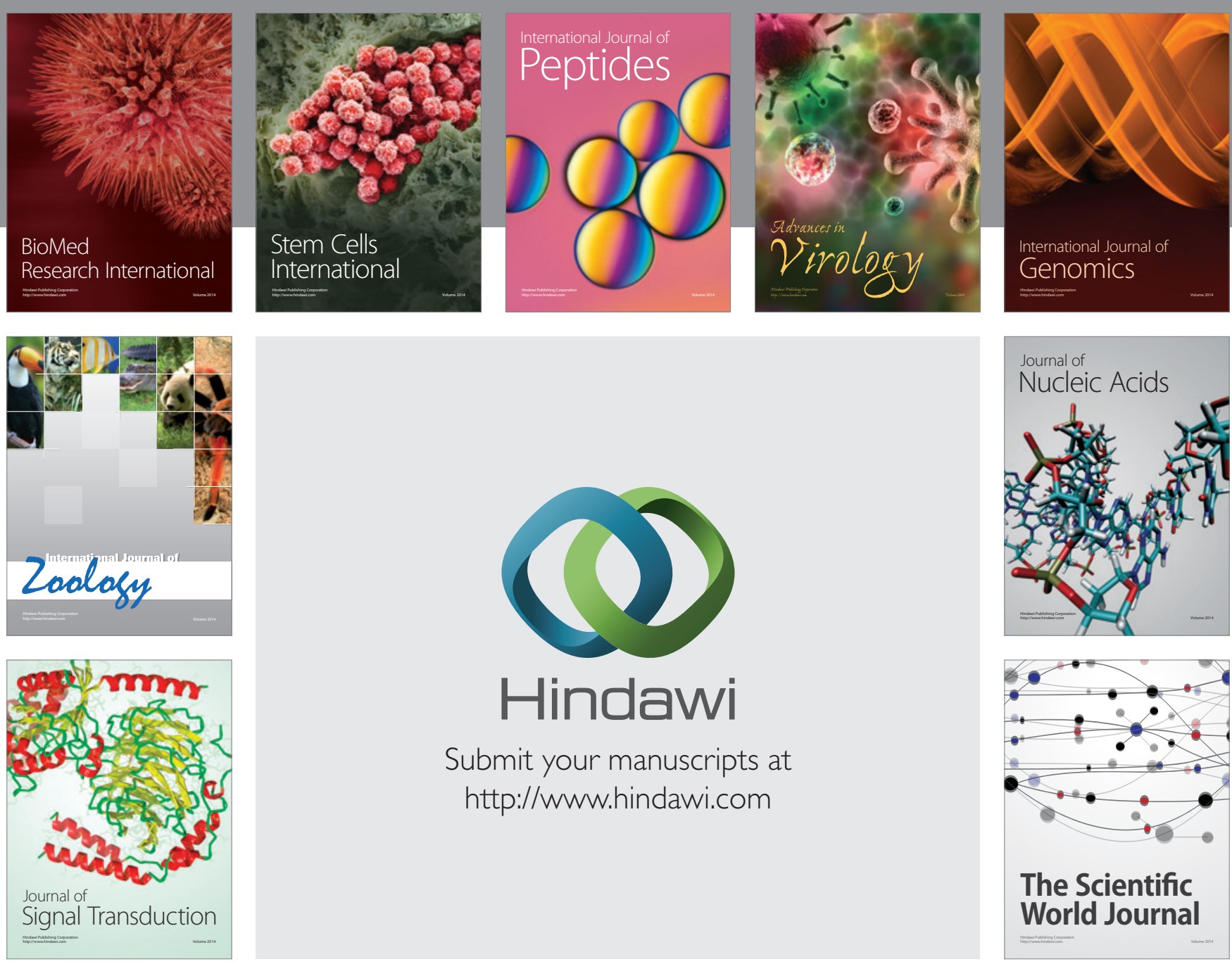

Submit your manuscripts at

http://www.hindawi.com
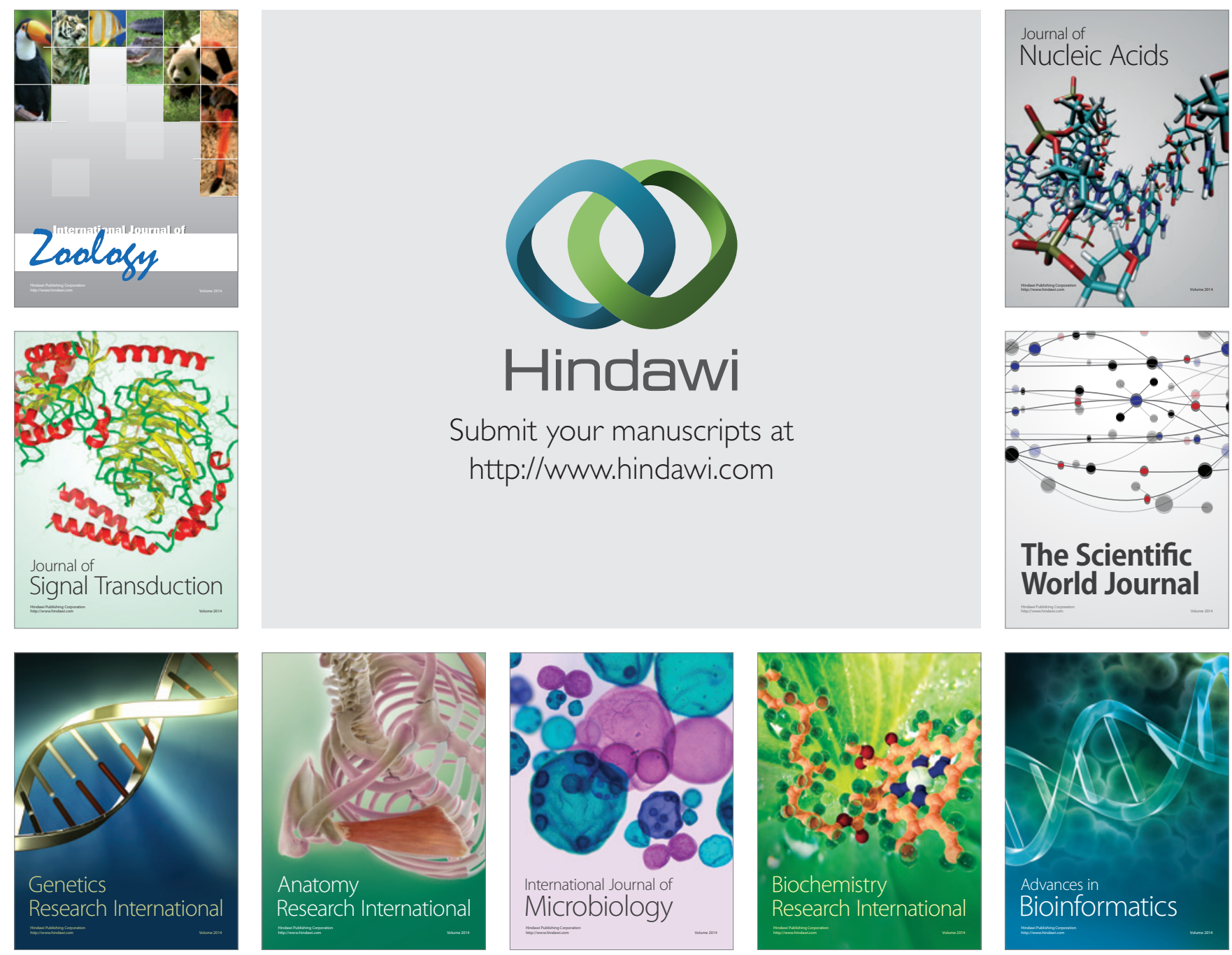

The Scientific World Journal
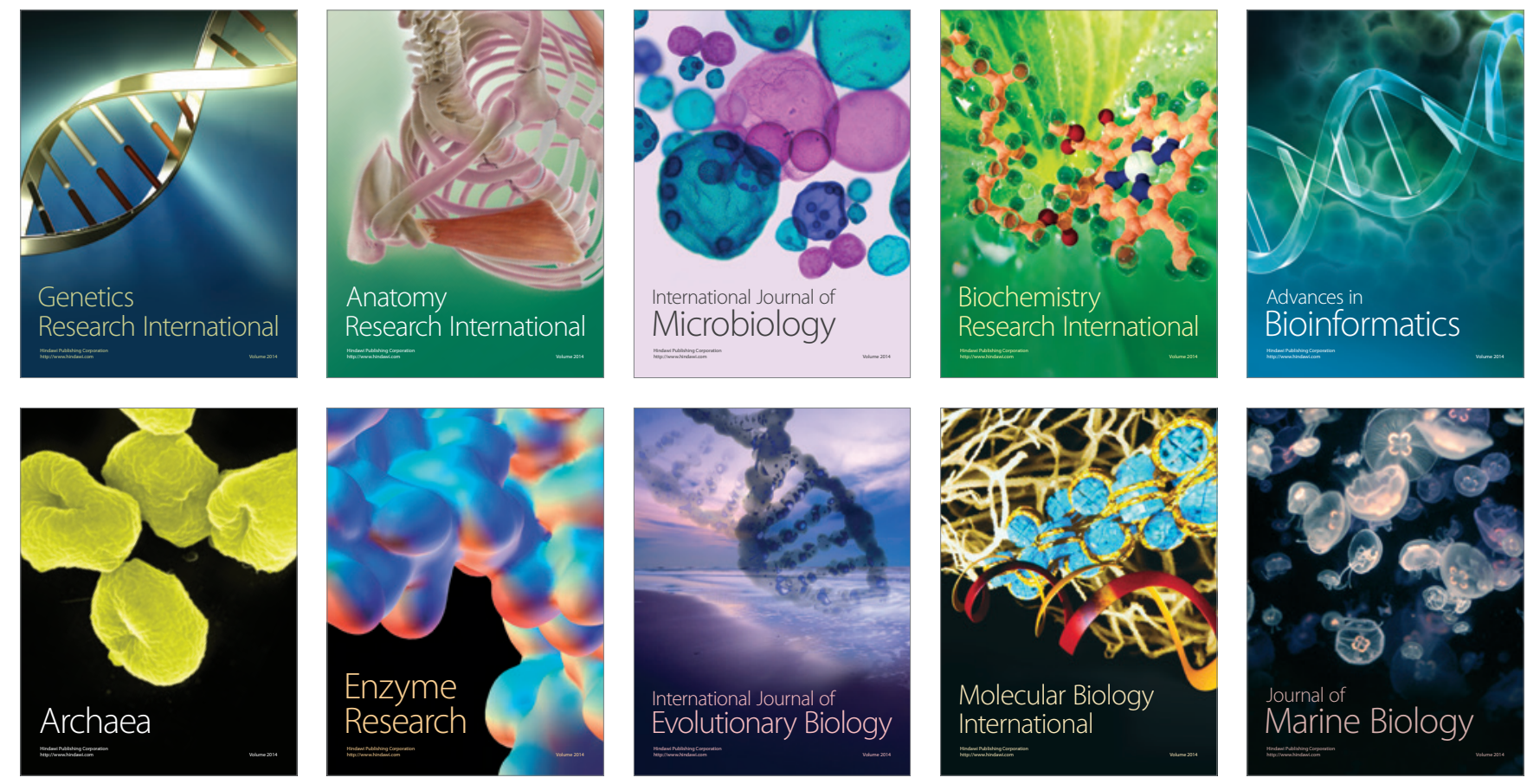